\title{
A COMPARISON OF L-BAND AND C-BAND MULTIPATH PROPAGATION AT EDWARDS AFB
}

\author{
Michael Rice and Michael Jensen \\ Brigham Young University \\ Provo, Utah, USA
}

\begin{abstract}
This paper summarizes L- and C-band propagation data based on multiple transmit and receive antennas in the flight-line environment at Edwards AFB. The data show that for this particular environment, C-band propagation exhibits much less delay spread than L-band propagation. But C-band propagation is more susceptible to complete outages due to shadowing. The main contributing factors to these conclusions are the increased attenuation at the point of reflection at C-band and the fact that the same antennas were used for both the L- and C-band experiments. Consequently, the receive antenna beamwidths were different (the beamwidth was much narrower at C-band) and this narrowed the angular spread of the multipath components captured at C-band.
\end{abstract}

\section{INTRODUCTION}

In the context of aeronautical telemetry, understanding multipath propagation allows engineers to identify and evaluate the performance of multipath mitigation techniques. Because multipath propagation is the dominant channel impairment for low-elevation angle air-to-ground links, effective multipath mitigation has the prospect of improving the overall link availability. Whether or not a multipath mitigation technique can be identified as "effective" depends on the accuracy of the multipath propagation data used to test it. To this end, multipath propagation experiments have been conducted to collect data for the scenarios and frequency bands of interest.

This paper reports on multipath channel sounding experiments conducted along the flight line at Edwards AFB, California, in February 2011. The experiments were designed to address three important issues:

1. In its current form, the typical aeronautical telemetry system comprises an airborne transmitter and a ground station equipped with a large tracking antenna. The relatively narrow beamwidth of the ground-based receive antenna tends to attenuate off-boresite reflections in the propagation path for "up and away" flight profiles. However, low-elevation-angle 
and flight-line scenarios present serious challenges. Previous work for low-elevation-angle scenarios includes experiments conducted at the Air Force Flight Test Center, Edwards Air Force Base in L- and S-bands [1] and at Pt. Mugu Naval Air Station over the Pacific Ocean in X-band [2]. These experiments provided useful data for low-elevation-angle propagation in the "up and away" scenario at test ranges in the western United States. One shortcoming of these experiments, however, is the absence of flight-line propagation data.

2. Recent WRC allocations for aeronautical telemetry made the C-band frequencies 4400 $4900 \mathrm{MHz}, 5091-5150 \mathrm{MHz}$, and $5925-6700 \mathrm{MHz}$ available for augmenting the current L- and S-band allocations [3]. As a result, there have been efforts to develop RF hardware compatible with the new allocations [4] - [7] and some initial investigations into link performance at C-band [8]. Consequently, there is interest in understanding multipath propagation at C-band in typical aeronautical environments. Of particular interest is the how C-band propagation is the same and how $\mathrm{C}$-band propagation is different from $\mathrm{L}$ - and $\mathrm{S}$-band propagation.

3. Multipath mitigation techniques may be broadly categorized as diversity or equalization methods. Diversity techniques have had limited appeal in aeronautical telemetry due to cost - the expense of using more than one (expensive) tracking antenna to realize spatial diversity on the ground and the cost of additional bandwidth required to realize frequency and temporal diversity. Consequently, little work has been done to consider the impact of using spatial diversity. As a result, equalization techniques have received the most attention [9] - [15]. However, the emphasis in this prior work has been on blind and adaptive techniques, and the reported results present a relatively weak case for using such techniques in aeronautical telemetry. This experience motivates the investigation of multiple antenna techniques that combine diversity and equalization to overcome the multipath interference.

The experiments described in this paper were intended to "plug some of the holes" in our understanding of RF propagation in aeronautical telemetry. Collecting L- and C-band data from the same transmitters and receivers in the same locations facilitates side-by-side comparisons using L- and $\mathrm{C}$-band data. This helps the community understand how $\mathrm{C}$-band propagation is the same and different from L-band propagation. For the first time, multipath propagation data is collected along the flight line. This data will improve our understanding of multipath propagation in one of the most difficult scenarios test engineers usually encounter and help in identifying mitigation techniques that make reliable communication possible. Finally, multiple transmit antennas on the aircraft and multiple receive antennas on the ground are used to provide data relevant for the analysis of both equalization and spatial diversity mitigation techniques.

We show that in the flight line environment at Edwards AFB, multipath propagation is more pronounced at L-band than at C-band, but that C-band is more prone to signal outages due to shadowing than L-band. 


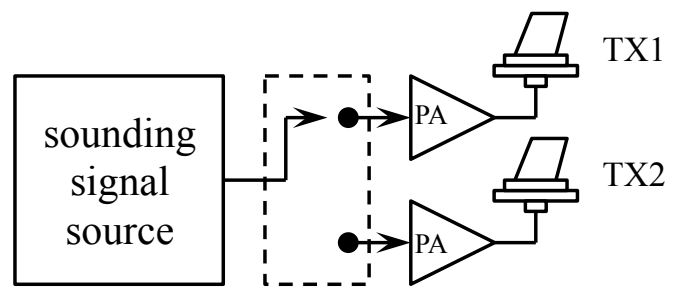

RF switch

Figure 1: A block diagram of the transmitter system used for the channel sounding experiments.

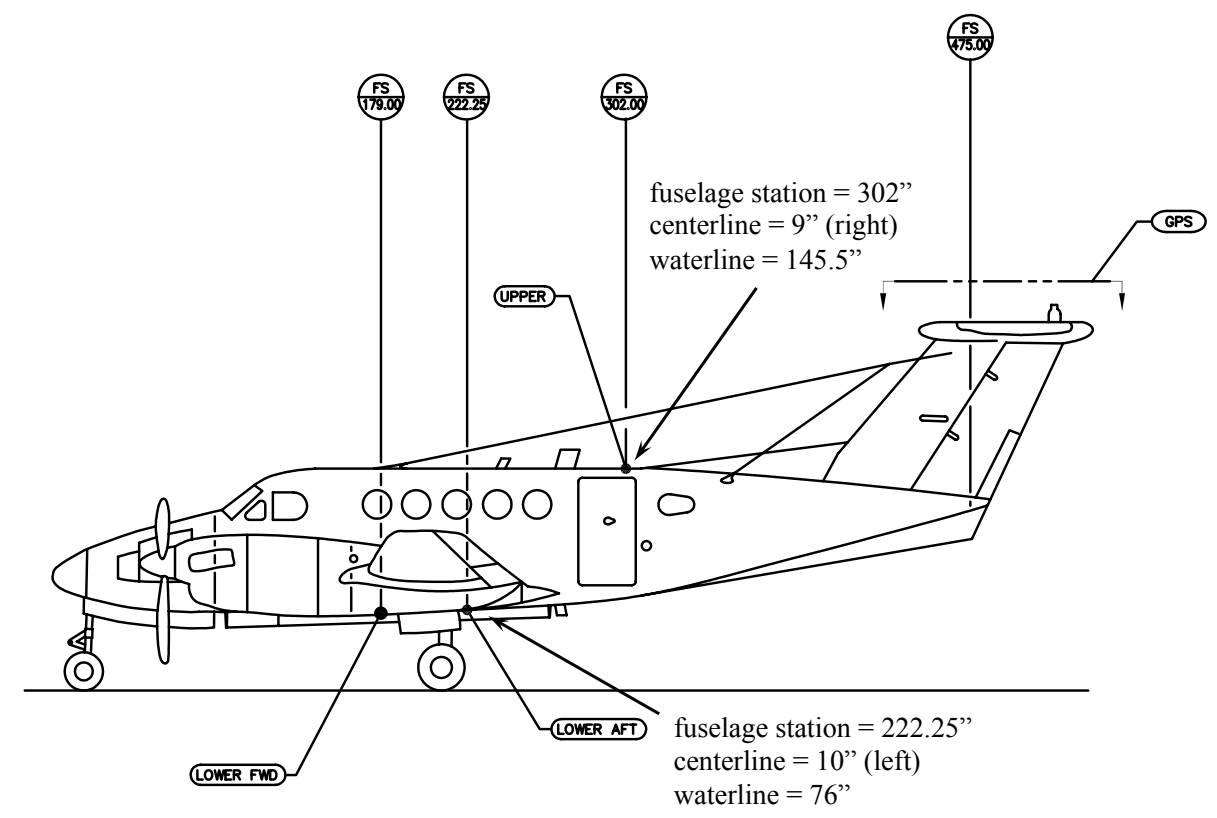

Figure 2: A diagram of C-12 aircraft showing the locations of the two antennas used for the channel sounding experiments.

\section{EXPERIMENTAL CONFIGURATION}

\section{A. System Configuration}

A block diagram of the transmitter for both the L- and C-band experiments is illustrated in Figure 1. The channel sounding signal comprised 501 unmodulated tones with a $100-\mathrm{kHz}$ spacing. The occupied bandwidth was $50 \mathrm{MHz}$. The RF channel sounding signal was applied to a Herley F9140W RF switch as shown. The dwell time for each switch position was $50 \mu$ s (5 periods of the channel sounding signal). The two switch outputs were connected to separate RF power amplifiers each cabled to a transmit antenna. The transmit antennas were small blade antennas (UB Corp. AO4459) mounted on the fuselage of the C-12 as illustrated in Figure 2. For the L-band experiments, the channel sounding signal was centered at $1824.5 \mathrm{MHz}$ (in upper L-band) and $10 \mathrm{~W}$ Remec power amplifiers were used. For the C-band experiments, the channel sounding signal was 


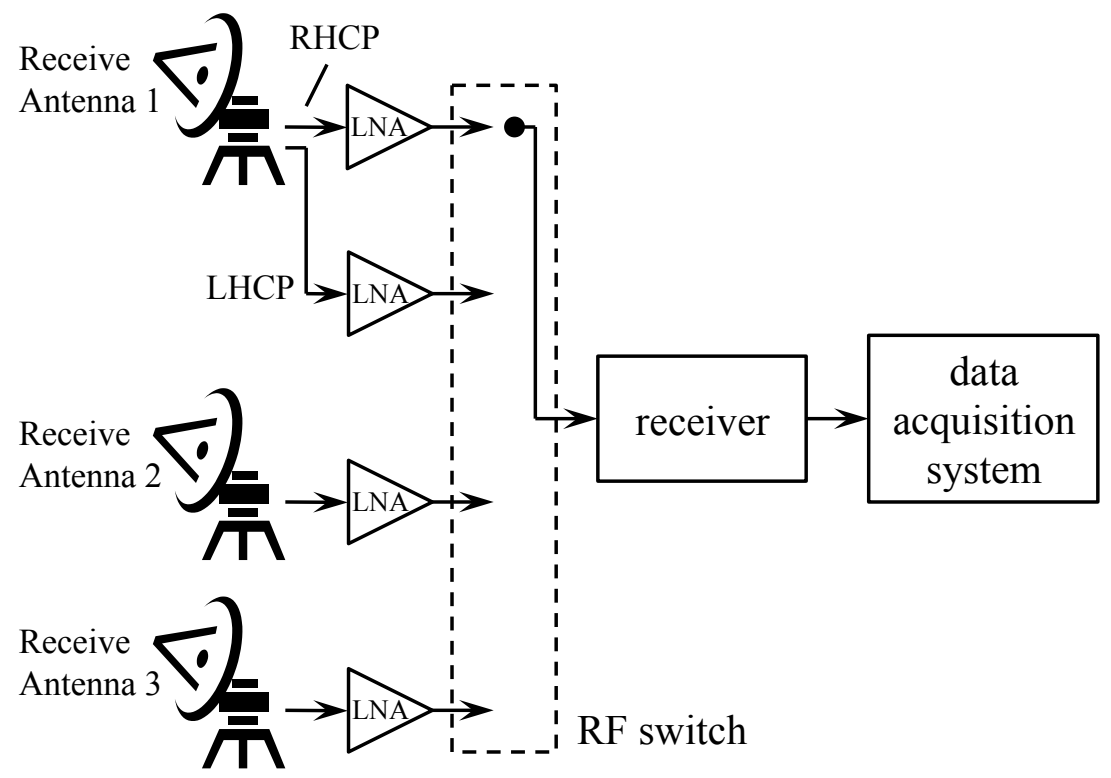

(a)

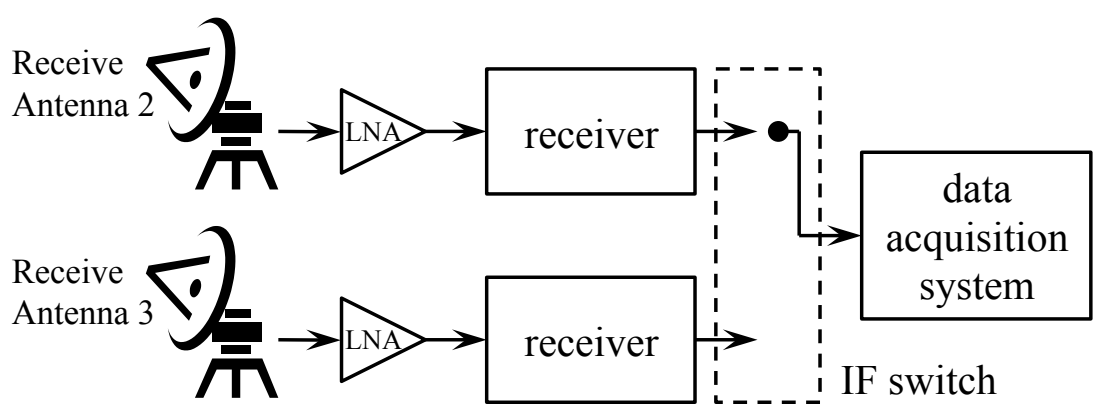

(b)

Figure 3: Block diagrams of the ground station configurations used for the channel sounding experiments: (a) the system used for the L-band experiments (1824.5 MHz); (b) the system used for the C-band experiments (5124 MHz).

centered at $5124 \mathrm{MHz}$ and 50-W Aethercomm (model SSPA 2.506.0-50) power amplifiers were used. In both cases, the power amplifiers were backed off to meet spectral occupancy regulations.

The ground station (receiver) was located at Building 4795 at Edwards AFB. Different ground station configurations were used for the channel sounding experiments at L- and C-bands. The ground station configuration used for L-band is illustrated in Figure 3 (a). Here, four receive antennas (based on three physical antennas) were used. The relative positions of the antennas is illustrated in Figures 4 and 5. Details of the antennas are summarized in Table 1. The RF outputs from the antennas were routed to a single receiver through an RF switch whose switching period was synchronized with the transmit switch using disciplined Rubidium oscillators. The dwell time for each receive antenna was $200 \mu \mathrm{s}$, which accommodated one $50 \mu$ s slot for each transmit antenna and one $100 \mu$ s blank period used for synchronization during data post-processing. The RF switch output was applied to a Cobham M/A-COM SMR-5550i microwave receiver, and the resulting 


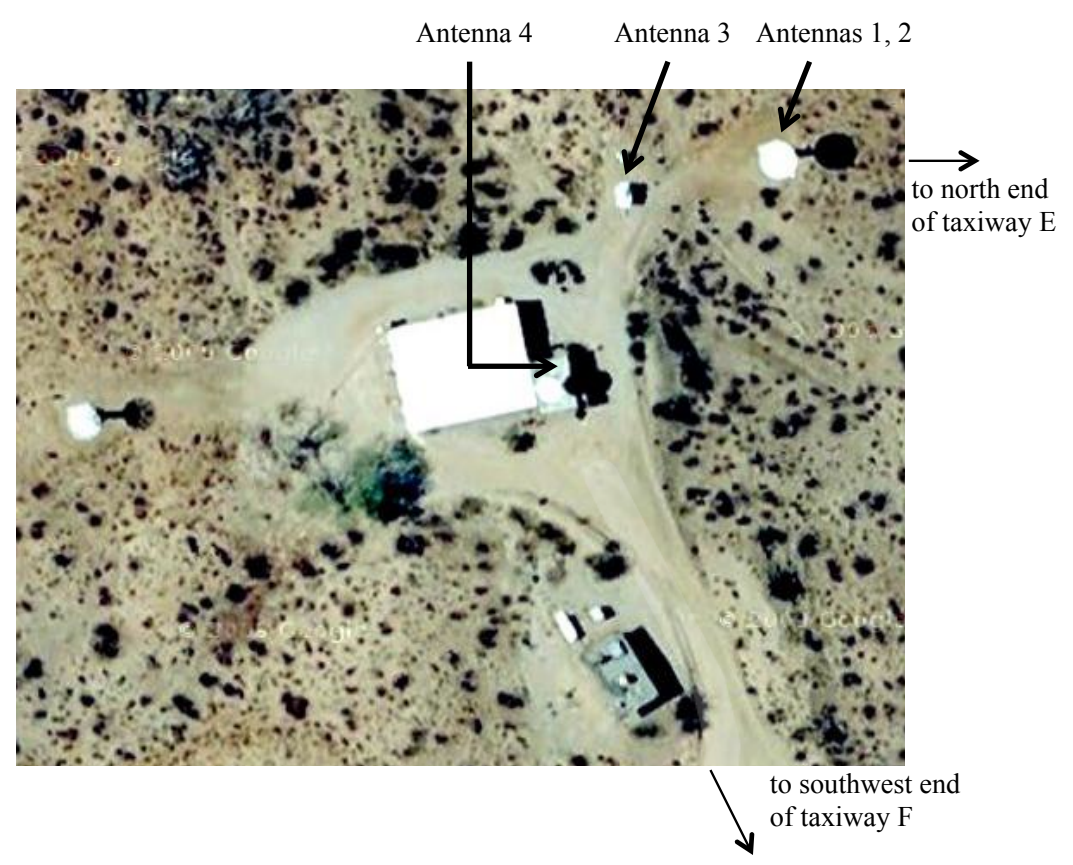

Figure 4: An aerial view of Building 4795 showing the positions of Antennas 1, 2, 3, and 4. All of the antennas were used for the L-band channel sounding experiments. Antennas 3 and 4 were used for the C-band channel sounding experiments.

intermediate frequency (70 MHz) signal was sampled at 200 Msamples/s by a Wideband Systems (DRS2200-144GB-2CHA1) data acquisition system and stored. GPS data were also recorded on the aircraft and incorporated into the post-processing.

The ground station configuration for the C-band experiments is illustrated in Figure 3 (b). Because a C-band feed was not available for Antennas 1 and 2, only Antennas 3 and 4 were used. Each antenna feed was downconverted to a $70 \mathrm{MHz}$ IF by separate Cobham M/A-COM SMR-5550i microwave receivers. The IF signals were applied a switch whose dwell time in each position was $200 \mu \mathrm{s}$ as explained in the previous paragraph. The switch output was applied to the data acquisition system. As before, GPS data were recorded on the aircraft and incorporated into the post-processing.

\section{B. Experimental Environment}

The $\mathrm{C}-12$ taxied along taxiways $\mathrm{E}$ and $\mathrm{F}$ at Edwards AFB. These taxiways are shown in the diagram of Figure 6. The relative positions of the transmitter locations (taxiways) and the receiver location (Building 4795) are shown on the map in Figure 7. Figure 8 shows the flight line from the roof of Building 4795. Observe that at the center of the image, the flight line is obscured from view by a small hill. Line-of-sight propagation between most of Taxiways $\mathrm{E}$ and $\mathrm{F}$ and Building 4795 is impossible because the hangers and buildings on the Building-4795-side of the taxiways. Not only do these structures block line-of-sight propagation, but provide ample opportunity for multiple 


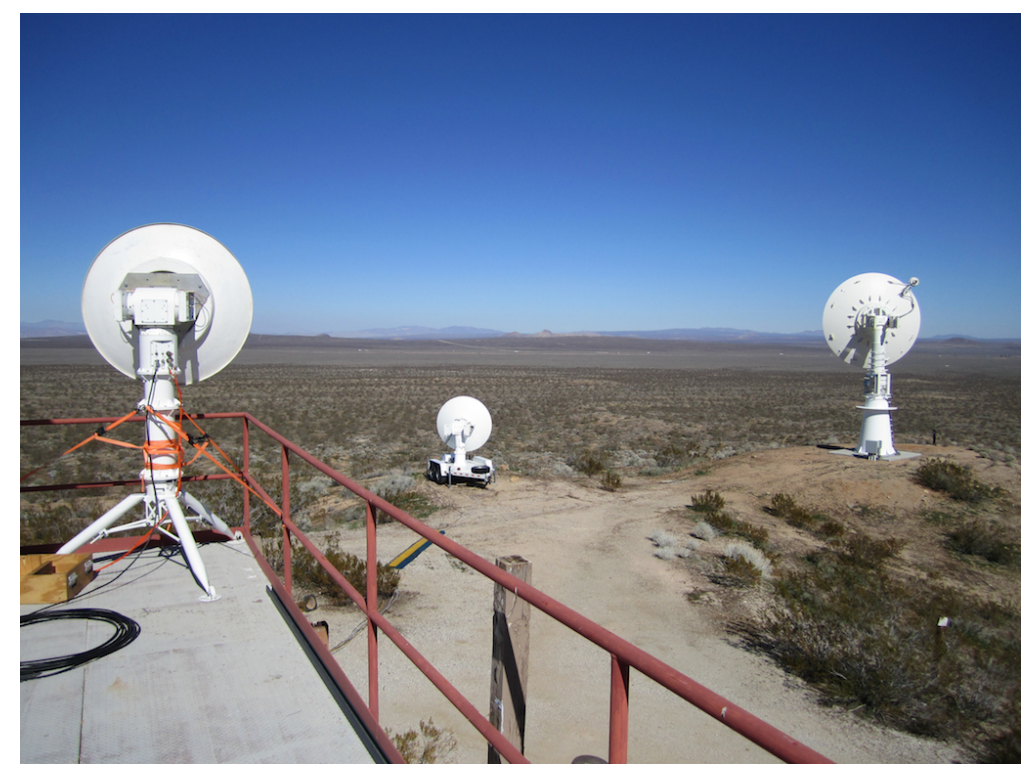

Figure 5: The view at Building 4795 showing the relative positions of the three physical antennas. Antenna 4 is in the foreground on the left, Antenna 3 is on the trailer in the middle, and Antennas 1 and 2 (aka "Antenna 5") is on the right. Note that in this image, the antennas are facing north, away from the flight line.

Table 1: Description of the receive antennas.

\begin{tabular}{|c|c|l|}
\hline Antenna & diameter & comments: \\
\hline RX1 & $5 \mathrm{~m}$. & $\begin{array}{l}\text { Building 4795 “Antenna 5" (EMT Model } \\
150), \text { tracking performed by conical scan, } \\
\text { RHCP. }\end{array}$ \\
\hline RX2 & $5 \mathrm{~m}$. & same as RX 1, except LHCP is used. \\
\hline RX3 & $8 \mathrm{ft}$. & tracking performed by conical scan, RHCP \\
\hline RX4 & $4 \mathrm{ft}$. & $\begin{array}{l}\text { tracking performed by steering using GPS } \\
\text { data downlinked from the C-12, RHCP }\end{array}$ \\
\hline
\end{tabular}



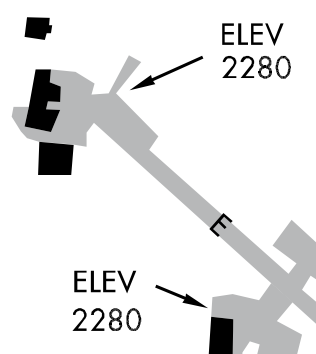

JUNE 2011

ANNUAL RATE OF CHANGE

$0.1^{\circ} \mathrm{W}$
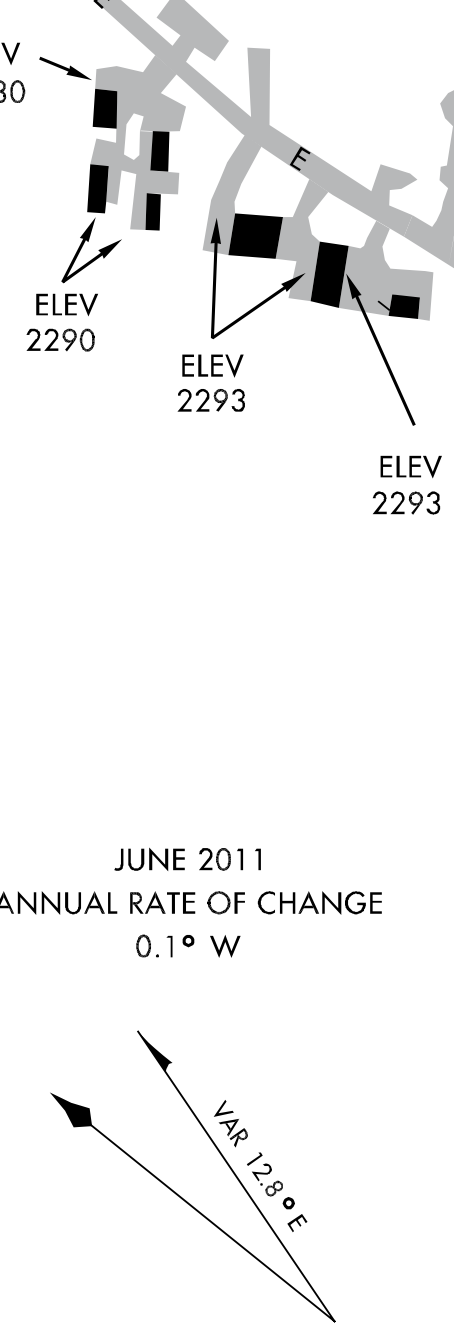

$\oplus$

0 2293

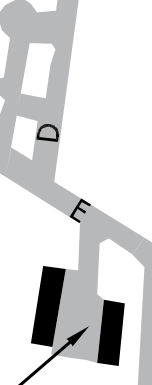

2280

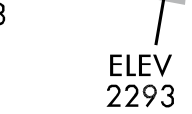

2463
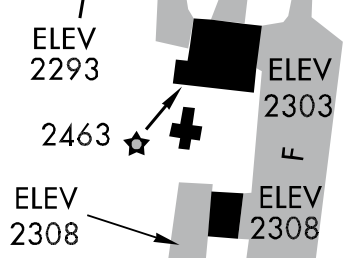

4

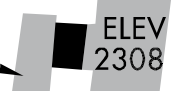

TOWER

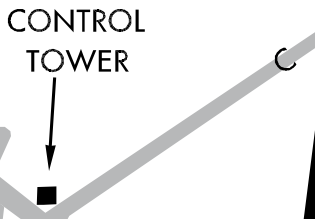

ELEV

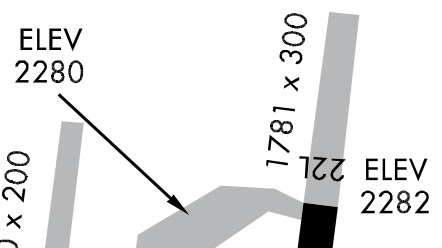

ELEV

2287

$8 y z z$

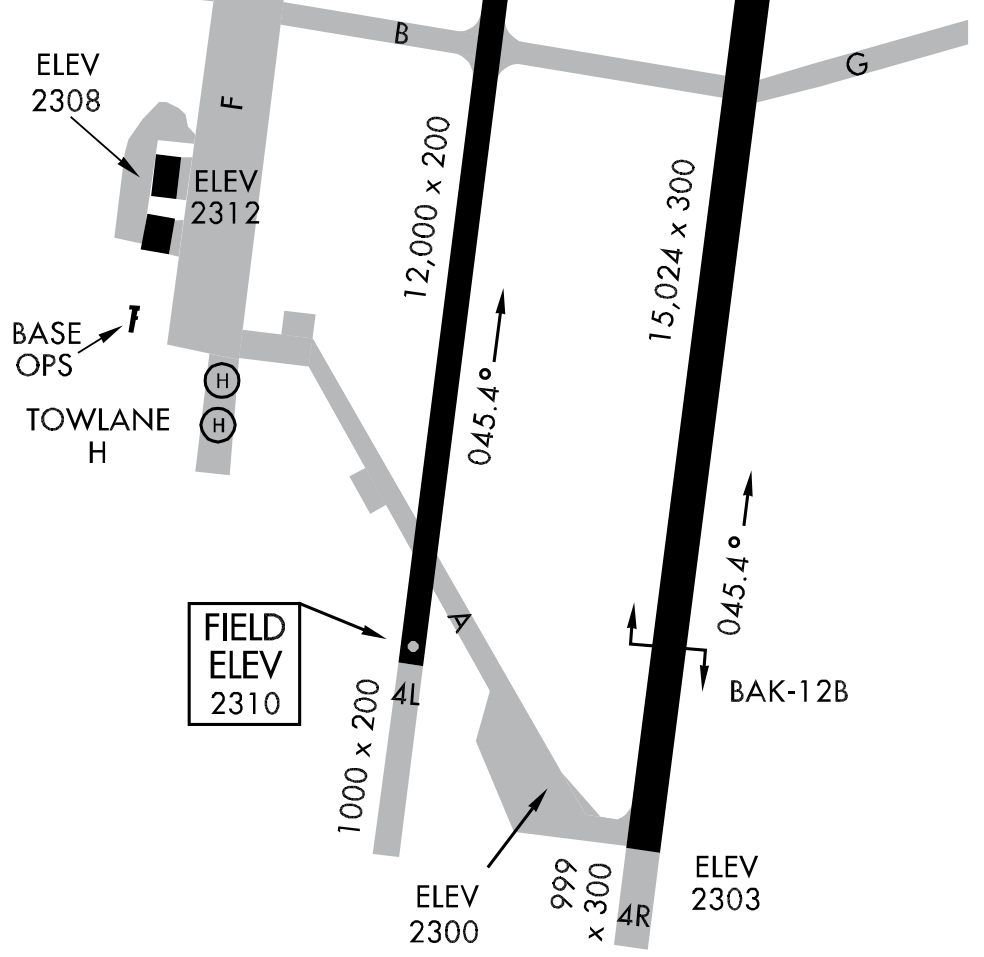

Figure 6: A diagram of the flight line at Edwards AFB, California, showing taxiways A - F (gray paths) and the runways (black paths). Modified from the FAA Edwards AFB airport diagram downloaded from http://aeronav.faa.gov/d-tpp/1107/00500AD.PDF 
propagation paths. It is this feature that presents such challenge to closing the telemetry link in these locations.

\section{Data Processing}

Samples of the band limited version of the channel impulse response were generated by examining the received signal over one sounding signal period of $10 \mu \mathrm{s}$. Denote by $s(t)$ the transmitted sounding signal by $r(t)$ the received signal. The relationship between the two is

$$
r(t)=s(t) * h(t)+w(t)
$$

where $h(t)$ is the unknown channel, $*$ is the convolution operation, and $w(t)$ is the additive thermal noise. The goal is to estimate samples of $h(t)$ from samples of one period of $r(t)$ and $s(t)$. Let $T$ be the sample time where $1 / T=200 \times 10^{6}$ samples/s and let $r(n T)$ and $s(n T)$ be the $n$-th sample of $r(t)$ and $s(t)$, respectively. Because there are $N=2000$ samples in a period, we have $0 \leq n<N$. The required deconvolution operation is performed in the frequency domain. Let $R\left(e^{j 2 \pi k / N}\right)$ and $S\left(e^{j 2 \pi k / N}\right)$ be the $k$-th sample of the length- $N$ DFT of $r(t)$ and $s(t)$, respectively, for $0 \leq k<N$. Then the $k$-th sample of DFT of the channel is

$$
\hat{H}\left(e^{j 2 \pi k / N}\right)=\frac{R\left(e^{j 2 \pi k / N}\right)}{S\left(e^{j 2 \pi k / N}\right)} .
$$

Notes:

1. The relationship $R\left(e^{j 2 \pi k / N}\right)=H\left(e^{j 2 \pi k / N}\right) S\left(e^{j 2 \pi k / N}\right)$ defines the frequency-domain relationship for the periodic extensions of $r(n T), h(n T)$, and $s(n T)$. As such, the corresponding time domain relationship $r(n T)=h(n T) * s(n T)$ is thought of as a circular convolution [16]. In general, the circular convolution and linear convolution are not equivalent. However, because the transmitted signal is periodic (and one period of the transmitted and received signals are used), the two convolutions are equivalent and (2) gives the desired result.

2. Because the samples $s(n T)$ define a real-valued bandpass signal, the division defined in (2) is only performed for the indexes corresponding to the region of support for $S\left(e^{j 2 \pi k / N}\right)$. Let this region of support be defined by the indexes $K_{1} \leq k \leq K_{2}$. Given the bandwidth, period, and sample rate, we have $K_{2}-K_{1}=500$. Consequently, there are 501 points in the region of support so that $\hat{H}\left(e^{j 2 \pi k / N}\right)$ is defined by 501 samples.

3. The discrete-time impulse response $\hat{h}(n T)$ is the inverse DFT of $\hat{H}\left(e^{j 2 \pi k / N}\right)$ for $K_{1} \leq k \leq$ $K_{2}$. The sample spacing for $\hat{h}(n T)$ is $5 \mathrm{~ns}$ (as determined by the sample rate).

\section{EXPERIMENTAL RESULTS}

\section{A. Normalizations and Calibrations}

In the following, $\hat{H}_{i j}^{(q)}\left(e^{2 \pi k / N}\right)$ represents the frequency response from the $j$ th transmit antenna to the $i$ th receive antenna at the $q$ th sample time. The corresponding discrete-time channel impulse 


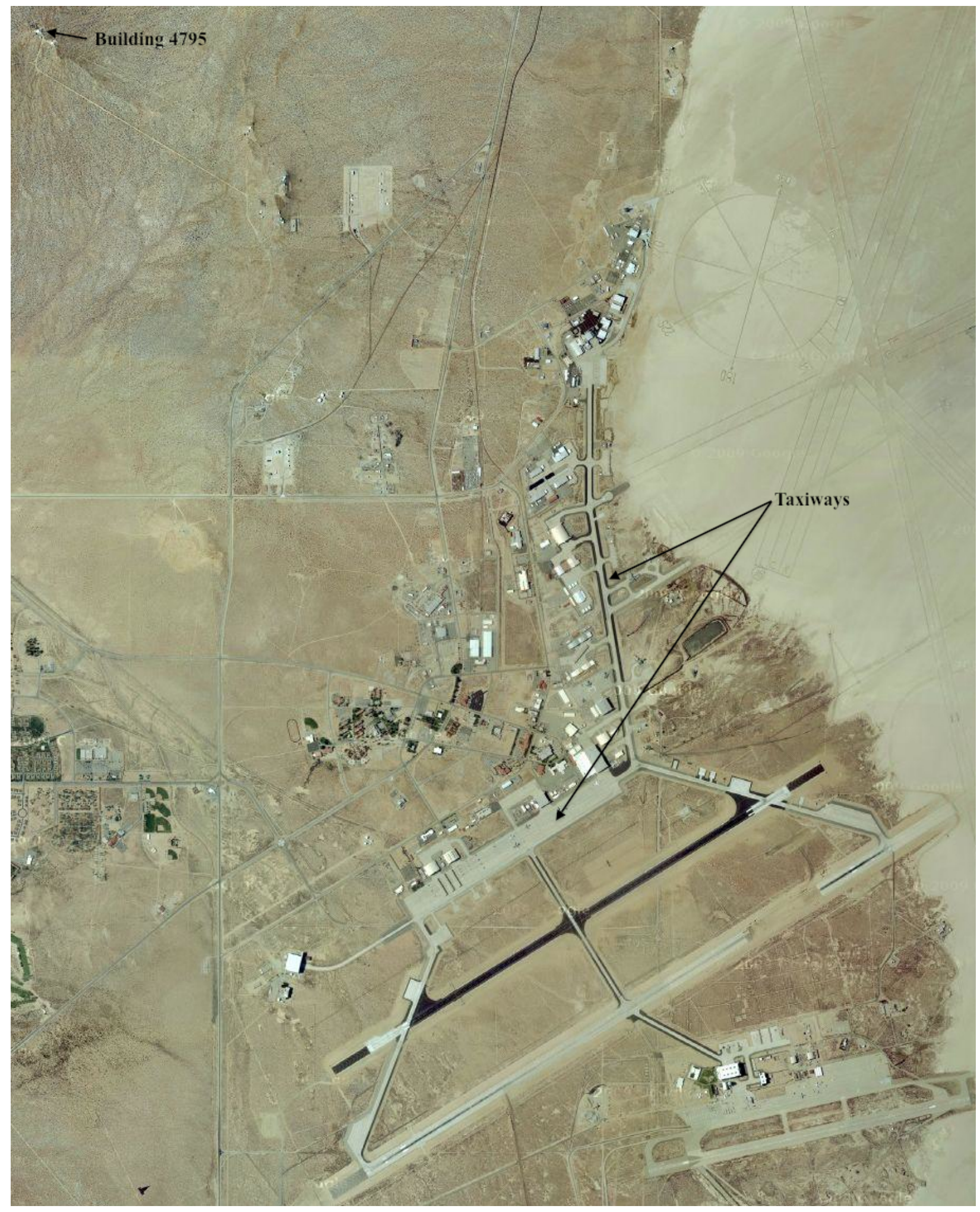

Figure 7: A map showing the relative positions of Building 4795 and the flight line at Edwards AFB. Building 4795 is in the upper left corner of the image. 


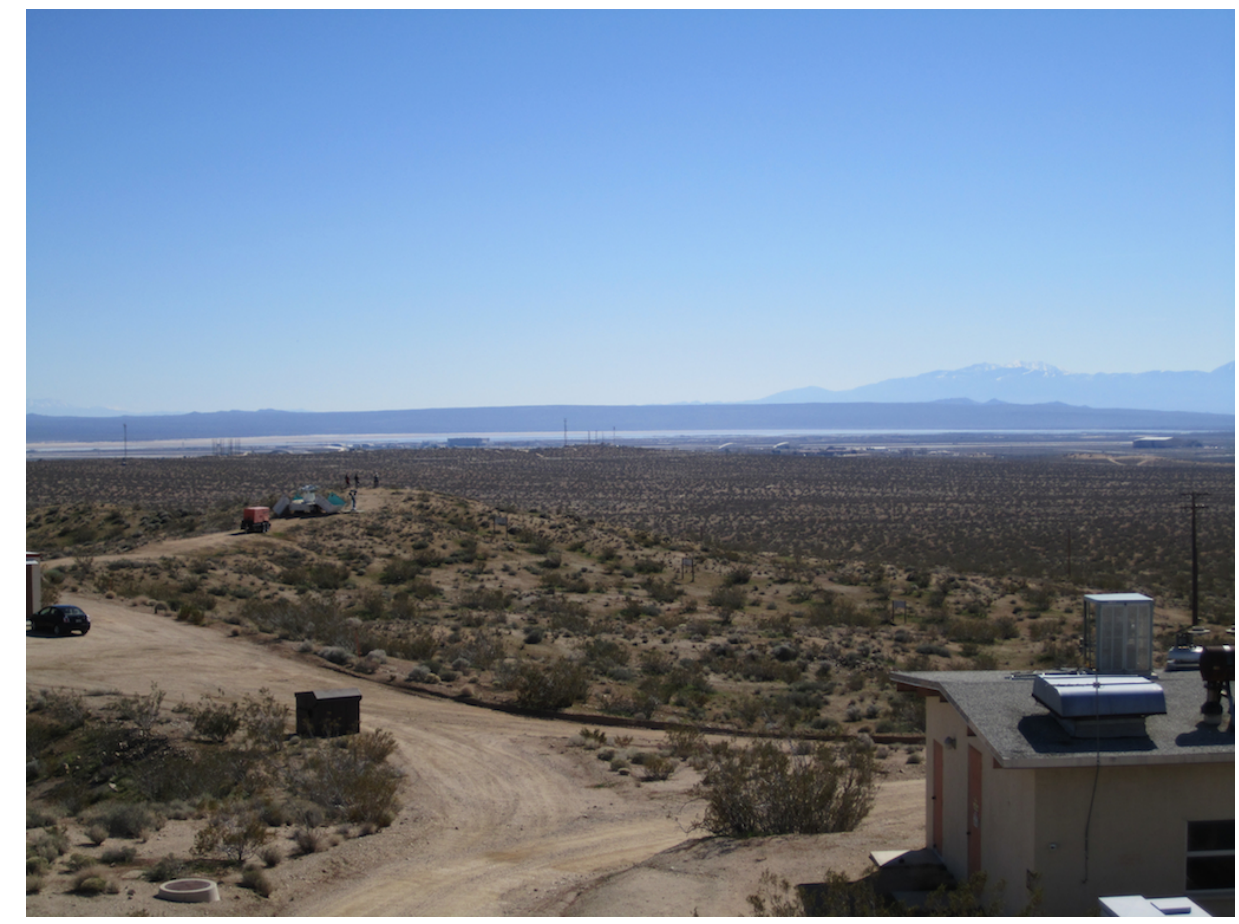

Figure 8: The flight line as seen from the roof of Building 4795 at the spot where Antenna 4 is located.

response is designated as $\hat{h}_{i j}^{(q)}(n T)$, where we emphasize that $n T$ represents the discretized delay variable.

The sampled channel frequency responses measured along the flight line for each pair of transmit and receive antennas are reduced to a sample rate of 1250 samples/second, providing a sampling in spatial displacement of the aircraft of approximately 30 samples per wavelength at $1824.5 \mathrm{MHz}$ and 11 samples per wavelength at $5124 \mathrm{MHz}$. Because of uncertainties regarding the responses of cables and electronics for each transmit and receive antenna, it is necessary to perform calibration on the observed channels before using them to analyze the channel behavior. This calibration involves power normalization and temporal alignment of the channel impulse responses for each antenna pair.

The power normalization must take into consideration the fact that the receive system uses automatic gain control (AGC) at the intermediate frequency, with the goal of ensuring that each received waveform has the same total received power. As the switches connect different antennas with varying gains, however, the AGC cannot always respond quickly enough to guarantee achievement of this goal. To remove the impacts of the notably different gains associated with the two receive antennas, we scale the responses observed on $\mathrm{RX} i$ by different values. Therefore, we compute the total power for the $q$ th channel transfer function from TX1 (the upper antenna) to receive antenna $i$, denoted respectively as $P_{\mathrm{T}, i}^{(q)}$ where $i \in\{1,2,3,4\}$ for the L-band experiments and $i \in\{3,4\}$ for the $\mathrm{C}$-band experiments as explained above. We then normalize the channel transfer functions as $\hat{H}_{i j}^{(q)} \leftarrow \hat{H}_{i j}^{(q)} / \sqrt{P_{\mathrm{T}, i}^{(q)}}$ (functional notation dropped). This preserves the rel- 
ative scaling between responses for different transmit antennas, since these gains are dictated by antenna placement and not by differences in antenna gain.

With the gains properly normalized, we compute the impulse responses using an inverse DFT. Differences in delays observed in the responses from different transmit antennas are due to different cable lengths to each antenna as well as the different positions of the antennas on the airframe, and these differences are small compared to the delay spreads observed in the multipath channels. In contrast, differences in delays observed in the responses from different receive antennas are significant due to different electronic subsystems and the long cables used to connect these subsystems. Therefore, we align the responses observed on receive antenna $i$ using different temporal shifts. Specifically, for the channels between transmit antenna 1 and receive antenna $i$, we define the beginning of the channel impulse response as the first sample at which its magnitude reaches $20 \%$ of its peak value, denoted respectively as $n_{i}^{(q)}$. Using this definition reduces sensitivity to noise in the waveform and has been found to be robust for accurately detecting the beginning of each response. We then designate sample $n_{i}^{(q)}$ as zero delay for the two impulse responses observed on RXi.

\section{B. Power Delay Profiles}

Given this waveform calibration, we are prepared to examine the multipath delay structure. Specifically, we compute the power delay profile (PDP) of the impulse responses using [17]

$$
\operatorname{PDP}_{i j}(n T)=\frac{1}{Q_{\mathrm{m}}} \sum_{q=1}^{Q_{\mathrm{m}}}\left|\hat{h}_{i j}^{(q)}(n T)\right|^{2}
$$

where $Q_{\mathrm{m}}$ is the total number of temporal channel samples over the measurement. This PDP provides an average measure of the power as a function of delay in the multipath channel, and it both gives an indication of the type of fading that might be observed in typical channels and helps to define the complexity of the equalizer that can adequately compensate for the range of multipath delay signatures observed.

Figures 9-11 plot the PDPs for each transmit-receiver pair for Taxiway F, Taxiway E, and the Taxiway E turnaround, respectively. Several observations are immediately obvious from these figures.

1. Multipath more pronounced for L-band than for C-band, as evidenced by the long tail on the L-band PDPs but not on C-band PDPs. This is to be expected because the C-band multipath components are much weaker relative to the line-of-sight component because reflections at a higher frequency tend to experience more attenuation.

2. During the experiments, the authors' anecdotal observation is that the C-band signal was either very good or essentially non-existent during while the C-12 was on the taxiways. In contrast, the L-band signal was sometimes very good, sometimes distorted, and rarely non-existent during the taxiway experiments. This can be explained by the fact that the receive antennas possessed much narrower beams at $\mathrm{C}$-band than at L-band. This property, accompanied by the increased attenuation of reflections at $\mathrm{C}$-band mentioned in observation 
TX1
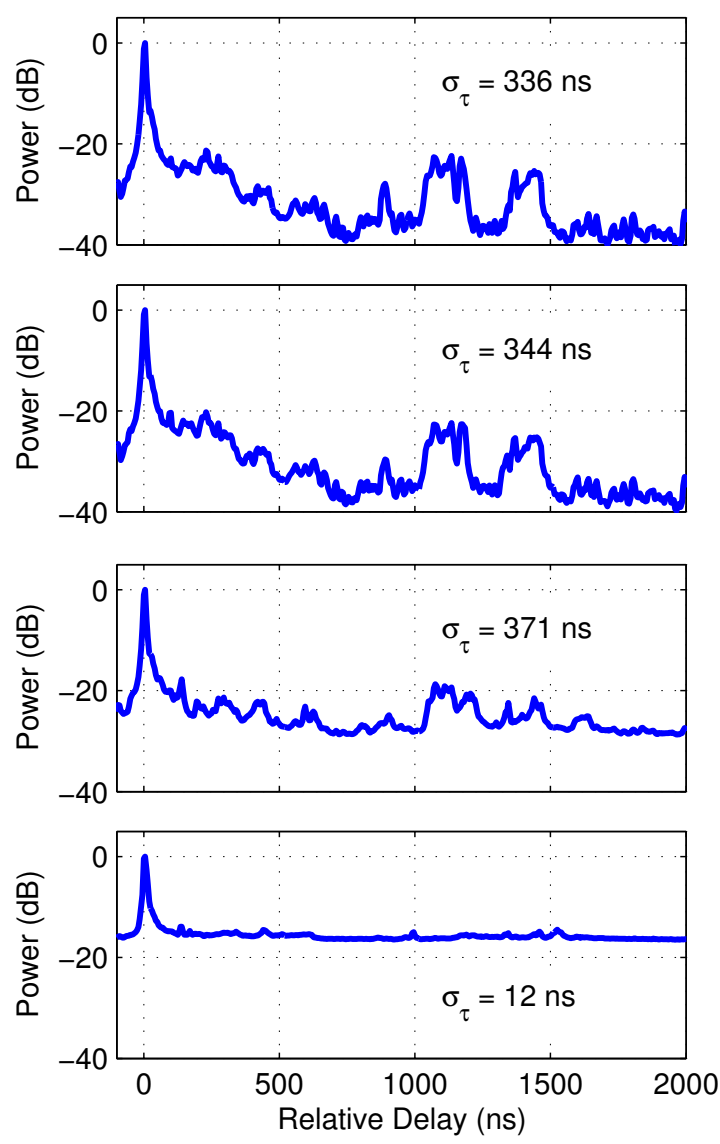

(a)
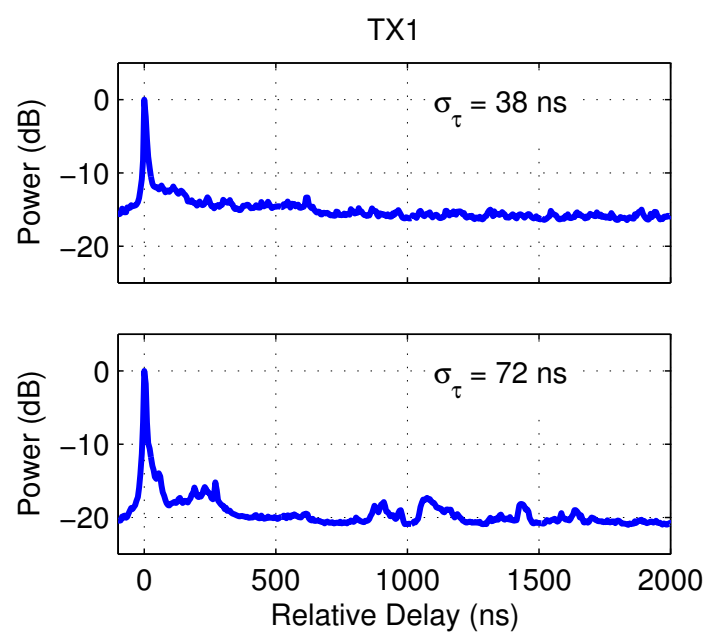

TX2

$\mathrm{RX} 1$

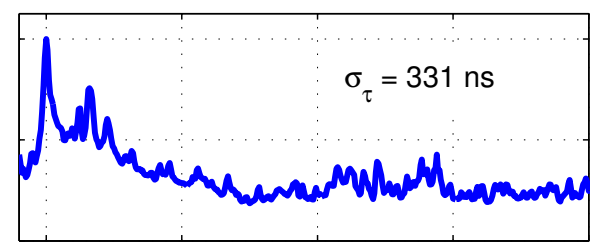

$\mathrm{RX} 2$

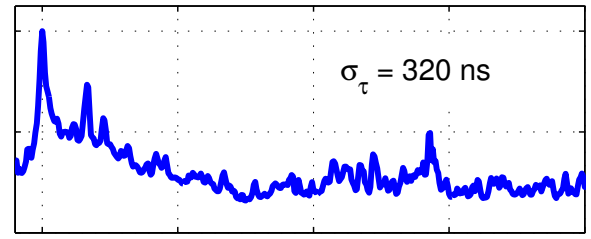

$\mathrm{RX3}$

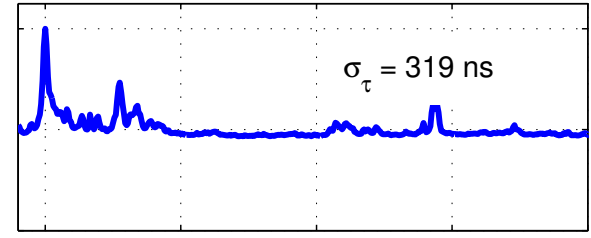

RX4

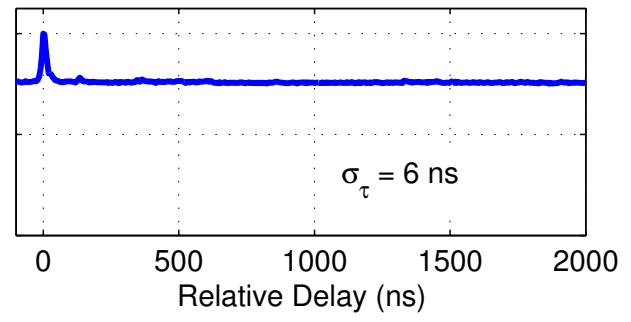

TX2

$\mathrm{RX3}$

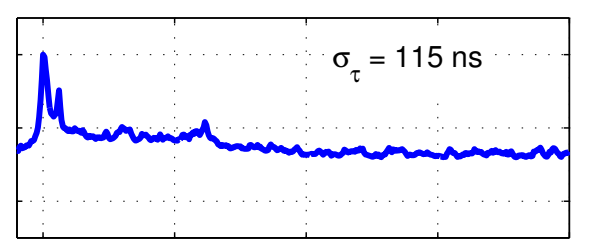

$\mathrm{RX} 4$

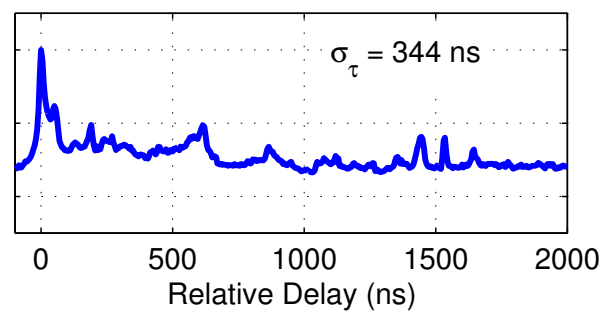

(b)

Figure 9: Power delay profiles for Taxiway $\mathrm{F}$ as the $\mathrm{C}-12$ taxis from southwest to northeast: (a) L-band PDPs, (b) C-band PDPs. 
TX1
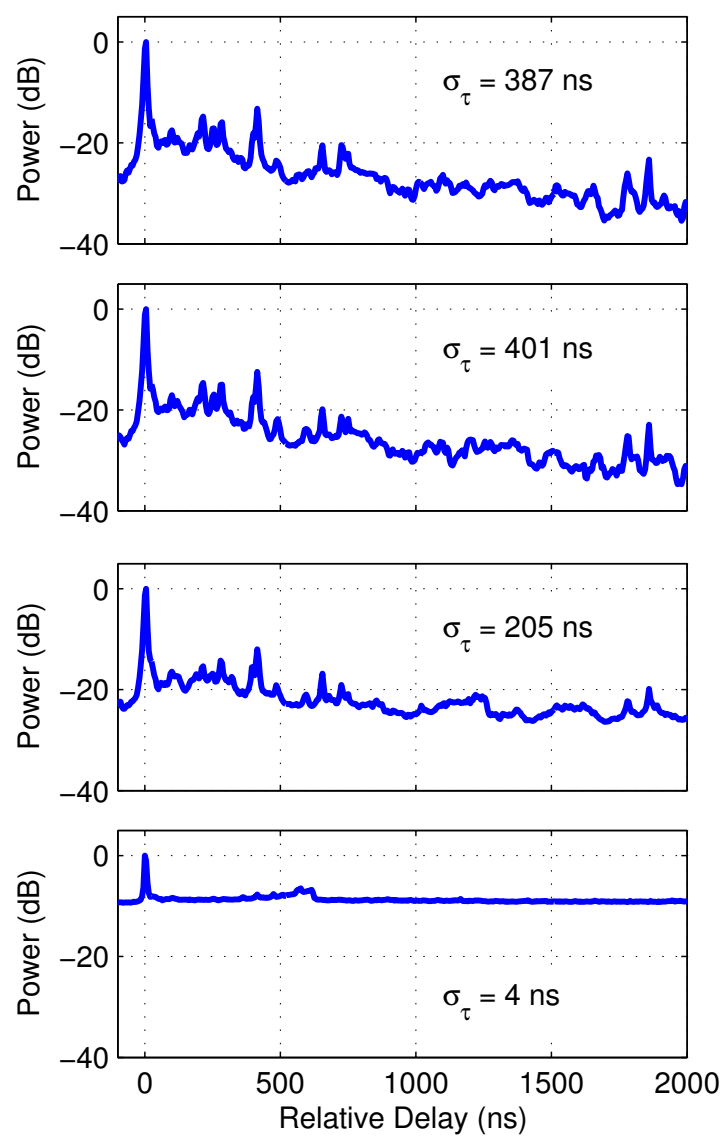

(a)
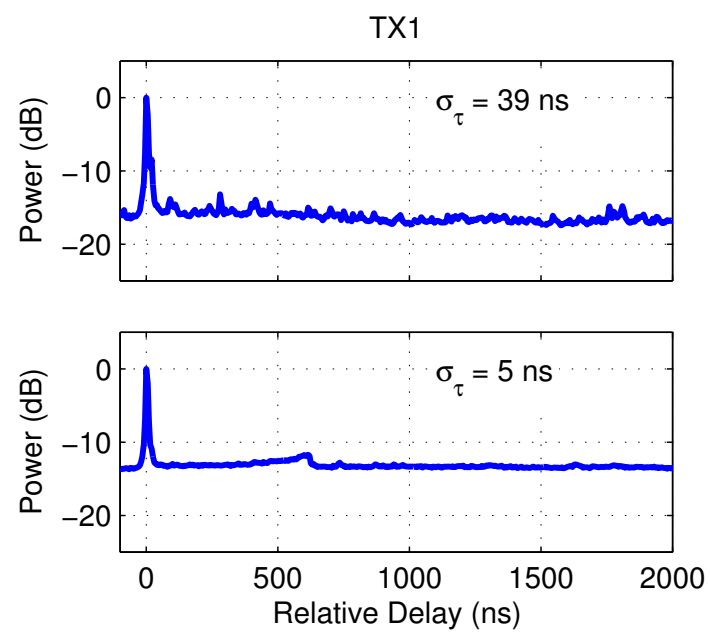

TX2

$\mathrm{RX} 1$

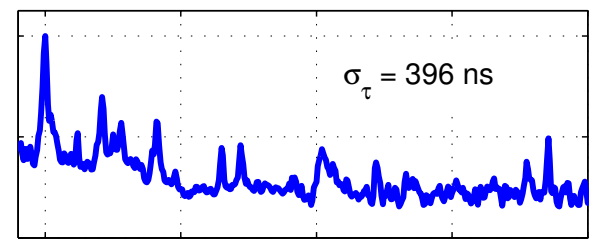

$\mathrm{RX} 2$

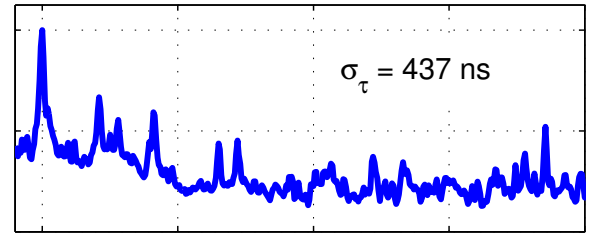

$\mathrm{RX3}$

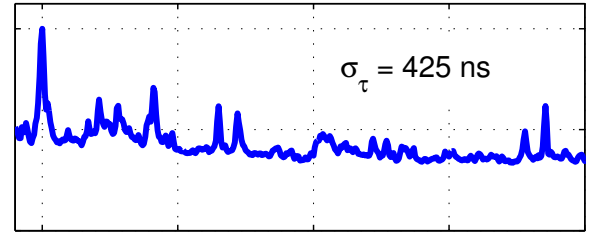

RX4

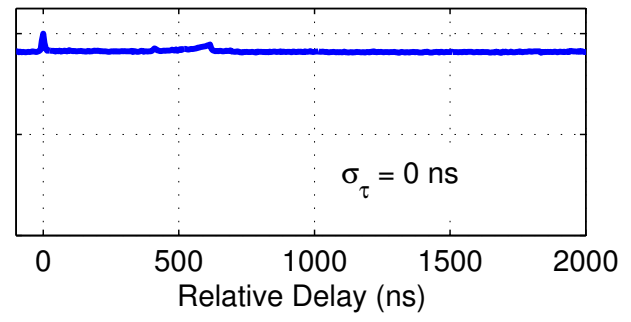

TX2

$\mathrm{RX3}$

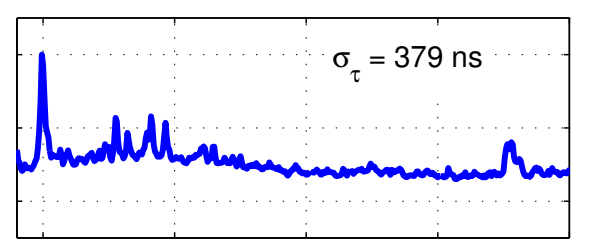

$\mathrm{RX} 4$

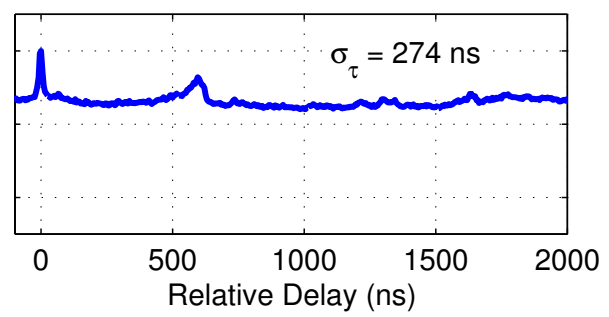

(b)

Figure 10: Power delay profiles for Taxiway E as the C-12 taxis from south to north: (a) L-band PDPs, (b) C-band PDPs. 
TX1
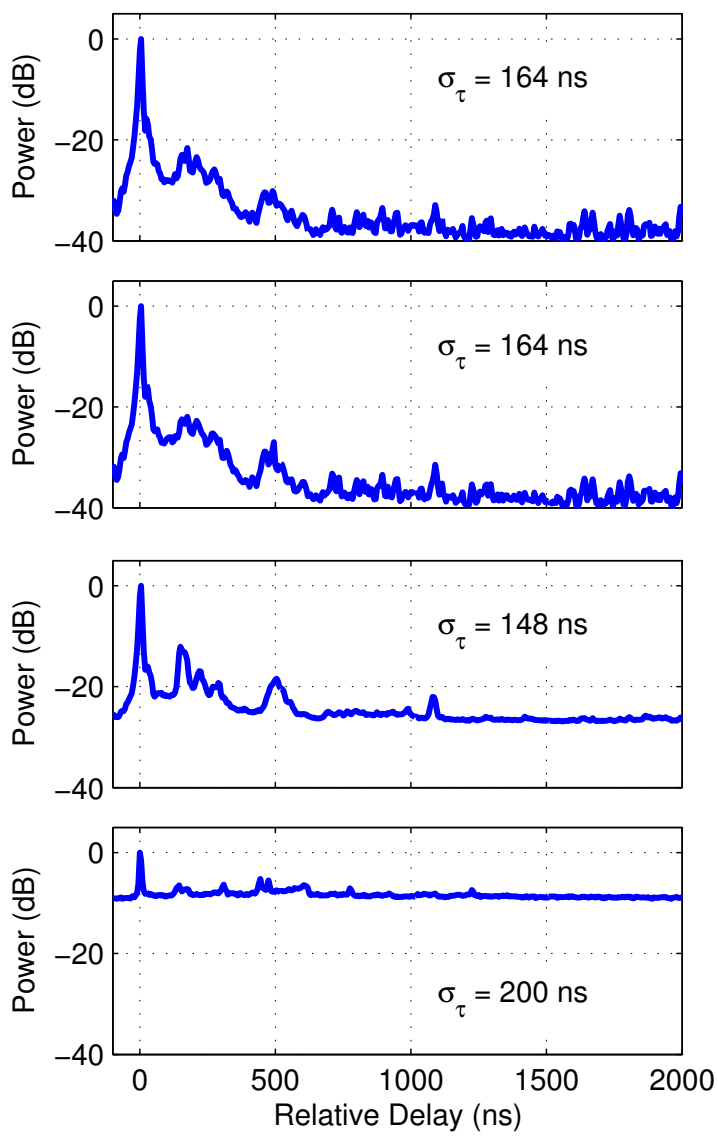

(a)
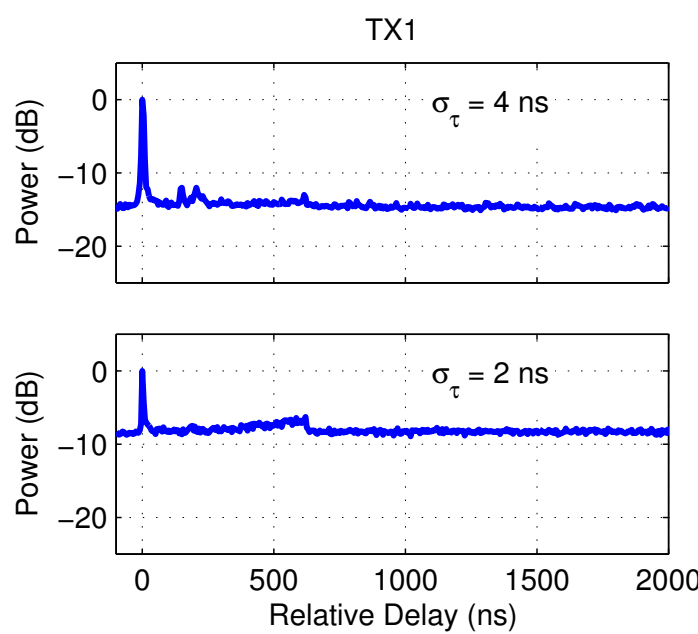

TX2

$\mathrm{RX} 1$

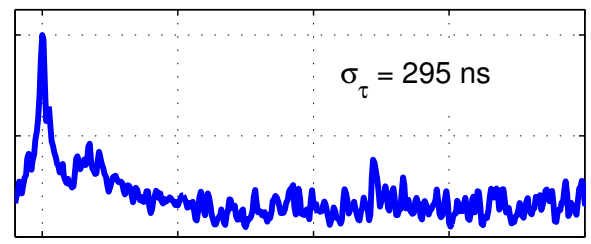

$\mathrm{RX} 2$

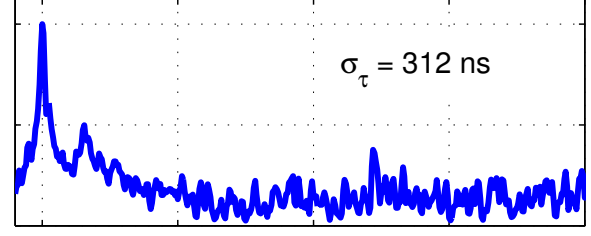

$\mathrm{RX3}$

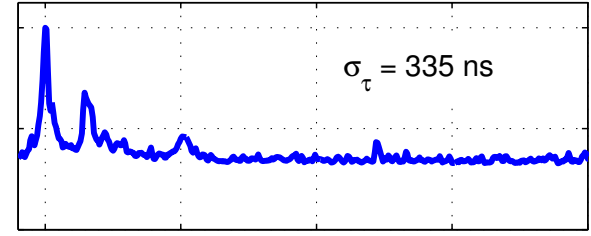

$\mathrm{RX} 4$

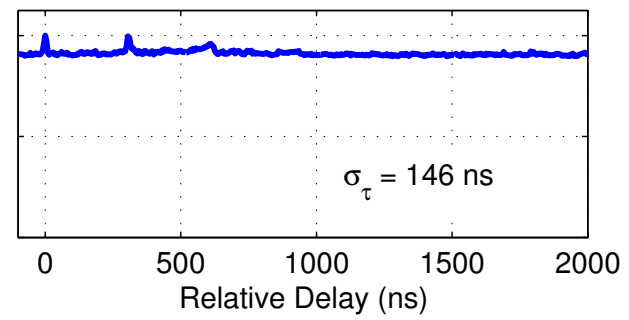

TX2

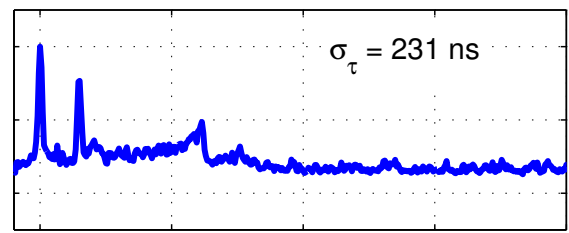

$\mathrm{RX} 4$

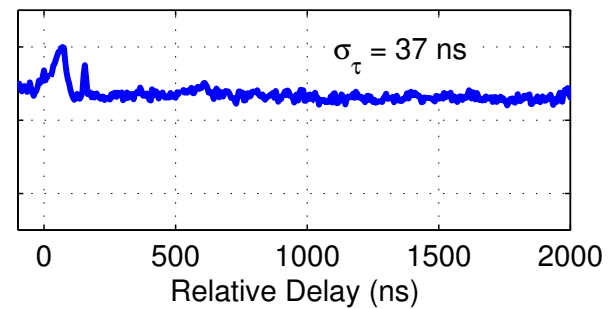

(b)

Figure 11: Power delay profiles for the north end of Taxiway $\mathrm{F}$ as the C-12 turned around: (a) L-band PDPs, (b) C-band PDPs. 
1, generate a "cleaner" multipath environment at C-band than at L-band. The reader should be aware that these observations only apply to the taxiway scenario. In other environments, these differences may not be as pronounced.

3. The PDPs for receive antennas 1 and 2 (L-band only) are, for all practical purposes, identical. This is because the signal is received by the same physical antenna but with different polarizations. The average channel is polarization independent. It remains to be seen if polarization impacts the instantaneous channel.

4. Receive Antenna 4 performs very poorly at L-band, but gives acceptable performance at $\mathrm{C}$-band. The L-band behavior is most likely due to the fact that the receive antennas use different tracking mechanisms. Receive antennas $1-3$ use a conical scan to maximize the received signal. Therefore, if the LOS path is obscured, receive antennas $1-3$ likely will track on a strong multipath component, and with the higher gain and narrower beamwidth will be less likely to see other multipath components. In contrast, receive antenna 4 tracks by pointing to the GPS coordinates of the aircraft (communicated through a dedicated telemetry link). This means it will point at the aircraft regardless of the quality of the LOS signal, possibly resulting in reduced overall power but richer multipath observations. In contrast, Cband propagation is "cleaner" as explained in observation 2 . The absence of strong multipath components means all antennas tend to point in the same direction. This renders the PDPs more similar than different.

5. Transmit antenna 2 is clearly inferior on the taxiways. (This is evident by the higher "floor" on the PDPs.) This is because it is located on the bottom of the $\mathrm{C}-12$ and the receive antennas are looking down on the $\mathrm{C}-12$.

6. These PDP plots provide a visual indication of the extent of delay experienced for the observed channels. However, we can further quantify this delay extent using the average delay spread for each channel. The average delay spread $\sigma_{\tau, i j}$ for each PDP can be computed using [17]

$$
\sigma_{\tau, i j}^{2}=\frac{1}{P_{i j}} \sum_{n}(n T)^{2} \operatorname{PDP}_{i j}(n T)-\bar{\tau}^{2}
$$

where

$$
\begin{aligned}
P_{i j} & =\sum_{n} \operatorname{PDP}_{i j}(n T) \\
\bar{\tau} & =\frac{1}{P_{i j}} \sum_{n}(n T) \operatorname{PDP}_{i j}(n T) .
\end{aligned}
$$

The delay spread values are included in the PDP plots of Figures $9-11$. As can be observed, the responses average delay spread for C-band much smaller than for L-band when the signal-to-noise ratio is high. This is to be expected given observations 1 and 2. 


\section{SUMMARY AND CONCLUSIONS}

We have summarized L- and C-band propagation data using multiple transmit and receive antennas in the flight-line environment at Edwards AFB. The data show that for this particular environment, C-band propagation exhibits much less delay spread than L-band propagation. But C-band propagation is more susceptible to complete outages due to shadowing. The main factors influencing the differences between L- and C-band propagation are

1. The propagating electromagnetic wavefront is more severely attenuated during a reflection at higher frequencies. Consequently, the multipath reflections are weaker relative to the line-of-sight path (when it exists).

2. Because the same antennas were used for both the L- and C-band experiments, the receive antenna beamwidths were different (the beamwidth was much narrower at C-band) and this narrowed the angular spread of the weaker multipath components captured at C-band.

Differences with the performance of antenna 4 were due to the method used for tracking.

\section{ACKNOWLEDGEMENTS}

The authors acknowledge the hard work and contributions of Mr. Glen Wolf (Delphi Research, Inc., Air Force Flight Test Center) who put the system together and Mr. Kip Temple (Air Force Flight Test Center) who served as the flight test engineer.

This work was supported by the Test Resource Management Center (TRMC) Test and Evaluation Science and Technology (T\&E/S\&T) Program through a grant to BYU from the US Army Program Executive Office for Simulation, Training, and Instrumentation (PEO STRI) under contract W900KK-09-C-0016. Any opinions, findings and conclusions or recommendations expressed in this material are those of the author and do not necessarily reflect the views of the TRMC and T\&E/S\&T Program and/or PEO STRI. The Executing Agent and Program Manager work out of the AFFTC.

\section{REFERENCES}

[1] M. Rice, A. Davis, and C. Bettwieser. A wideband channel model for aeronautical telemetry. IEEE Transactions on Aerospace and Electronic Systems, 40(1):57-69, January 2004.

[2] Q. Lei and M. Rice. Multipath channel model for over-water aeronautical telemetry. IEEE Transactions on Aerospace and Electronic Systems, 45(2):735 - 742, April 2009.

[3] T. Chalfant. International consortium for telemetry spectrum (ICTS) report. In Proceedings of the International Telemetering Conference, San Diego, CA, October 2008. 
[4] T. Hamilton. Upgrade of RCB receivers for C-band reception. In Proceedings of the International Telemetering Conference, Las Vegas, NV, October 2009.

[5] W. Wang, D. Martz, and K. Hutzel. L-3 Communications 3rd generation telemetry transmitter, ST-5000 L/S/C band architecture and design efforts. In Proceedings of the International Telemetering Conference, San Diego, CA, October 2010.

[6] C. Oh, K. Lee, and S. Oh. Refraction effects for tracking error at C-band and S-band frequencies. In Proceedings of the International Telemetering Conference, San Diego, CA, October 2010.

[7] G. Blake, D. Shea, Y. Hoory, I. Krepner, J. Pein, and O. Nahshon. Multi-band (L/S/C) nested concentric cavity coaxial mode RF feed for auto-track telemetry systems. In Proceedings of the International Telemetering Conference, San Diego, CA, October 2010.

[8] K. Temple. Performance comparison of aeronautical telemetry in S-band and C-band. In Proceedings of the International Telemetering Conference, San Diego, CA, October 2010.

[9] R. Fan, K. Yao, and D. Whiteman. Adaptive equalization for OQPSK through a frequency selective fading channel. In Proceedings of the International Telemetering Conference, San Diego, CA, October 2000.

[10] Z. Ye, E. Satorius, and T. Jedrey. Enhancement of advanced range telemetry (ARTM) channels via blind equalization. In Proceedings of the International Telemetering Conference, Las Vegas, NV, October 2001.

[11] T. Hill and M. Geoghegan. A comparison of adaptively equalized PCM/FM, SOQPSK, and multi-h CPM in a multipath channel. In Proceedings of the International Telemetering Conference, San Diego, CA, October 2002.

[12] M. Geoghegan. Experimental results for PCM/FM, Tier I SOQPSK, and Tier II Multi-h CPM with CMA equalization. In Proceedings of the International Telemetering Conference, Las Vegas, NV, October 2003.

[13] E. Law. How well does a blind adaptive CMA equalizer work in a simulated telemetry multipath environment? In Proceedings of the International Telemetering Conference, San Diego, CA, October 2004.

[14] M. Rice and E. Satorius. A comparison of MMSE and CMA equalization techniques for ARTM Tier-1 waveforms. In Proceedings of the International Telemetering Conference, San Diego, CA, October 2004.

[15] M. Rice and E. Satorius. Equalization techniques for multipath mitigation in aeronautical telemetry. In Proceedings of the IEEE Military Communications Conference, Monterey, CA, November 2004.

[16] A. Oppenheim and A. Willsky. Signals and Systems. Prentice Hall, 1996.

[17] R. Vaughan and J. B. Andersen. Channels, Propagation, and Antennas for Mobile Communications. The Institution of Electrical Engineers, London, 2003. 